\title{
Nuclear spin relaxation in glassy ionic conductors
}

\section{S. R. ELLIOTT ${ }^{(1)}$}

Laboratoire de Science des Matériaux Vitreux, Université de Montpellier II, Place Eugène Bataillon, 34095 Montpellier Cedex 5, France

\begin{abstract}
The nuclear spin relaxation behaviour in disordered ionically conducting materials is ascribed to a mechanism based on diffusion-controlled relaxation. In this targetdiffusion model, relaxation is assumed to occur at interstitialcy sites, triggered by the arrival of diffusing ions. The dependence of the spin-lattice relaxation rate on the temperature, Larmor frequency and ionic concentration can all be understood with this model. The results of a nuclear spin-lattice relaxation study of the mixed-alkali effect in glasses can, on this basis, be interpreted in terms of changes in ionic mobility.
\end{abstract}

(1)Permanent address: Department of chemisty, University of Cambridge, Lensfield Road, Cambridge CB2 IEW, U.K. 


\section{Introduction}

The mechanism(s) of ionic transport in disordered (particularly glassy) materials remains ill-understood. Part of the problem lies with the fact that the local structural environment of the conducting ions is not known and, as a result, the conduction paths for the ions are not known either. Furthermore, normal experimental studies of ionic transport, such as d.c. conductivity measurements as a function of temperature or (total) ion concentration $\mathrm{N}$, do not provide direct information on the conduction process involved. Influences on the ionic conductivity $\sigma$ of possible changes in ionic mobility, $\mu$, or the mobile ion concentration, $n(\leq N)$, with variations in temperature or $\mathrm{N}$ need to be evaluated separately before it is possible to decide unequivocally between various models for the ionic transport mechanisms(s), such as those stressing the role played by the mobile ionic carrier concentration, $\mathrm{n}$ (for example in the "weakelectrolyte" theory [1]), or the ionic mobility [2] or "defects" such as interstitialcies (for a review, see ref [3]).

Studies of the dynamic behaviour of ionic transport processes in amorphous solids can be especially revealing; relevant techniques include frequency-dependent (a.c.) conductivity, quasi-elastic (neutron or light) scattering and, the subject of this paper, nuclear magnetic resonance (NMR) spin-lattice relaxation (SLR) rate. The goal is to formulate a microscopic model for the dynamic behaviour so that the proposed mechanism accounts satisfactorily for experimental data obtained from these (and related) measurements, encompassing a very wide range of frequencies (d.c. - $10^{11} \mathrm{~Hz}$ ).

\section{Nuclear spin relaxation in ionic glasses}

In recent years, a number of studies of nuclear spin relaxation in ionically conducting glasses have been reported, mainly concerned with the measurement of the SLR rate, $\mathrm{T}_{1}{ }^{-1}$, as a function of temperature or (Larmor) frequency, of various isotopes of atoms which, as ions, are mobile in glassy hosts; for recent reviews of this subject, see $[4,5]$.

The following general features appear to characterise the NSR behaviour of nuclei of diffusing ions in disordered ionically conducting materials. At sufficiently high temperatures (the upper limit being dictated by the glass-transition temperature) the temperature dependence of $\mathrm{T}_{1}^{-1}$ exhibits a peak which is asymmetric in shape. In an Arrhenius plot, the effective activation energies for the high- and low-temperature sides of the peak are usually very different; the high-temperature value, $E_{1}$, is comparable to that of the d.c. conductivity, $E_{\sigma}$ whereas, for not too low ion concentrations (see later), the low-temperature value, $E_{2}$, is considerably smaller:

$$
\mathrm{E}_{2}=\alpha \mathrm{E}_{\sigma}
$$

with $\alpha \approx 0.5$. A remarkable feature is that the parameter $\alpha$ is dependent on the ion concentration, $x$; as $x$ decreases towards zero, $\alpha$ tends to unity, i.e. the peak of SLR rate versus inverse temperature becomes symmetrical [6]. Furthermore, the slope of the high-temperature side of the peak of $\mathrm{T}_{1}^{-1}$ versus inverse temperature is independent of Larmor frequency (or equivalently magnetic field) in the motional-narrowing limit; that of the low-temperature side, in contrast, is frequency dependent with an approximate power-law frequency dependence, $\mathrm{T}_{1} \propto \omega \mathrm{m}$, where the exponent $\mathrm{m}$ may be slightly sub-linear or somewhat super-linear but, in all cases, $0<m<2$. 
At the lowest temperatures, $T \leq 200 \mathrm{~K}$, the temperature dependence of the SLR rate becomes much weaker and can be represented as a power law, $\mathrm{T}_{1}{ }^{-1} \propto \mathrm{T}^{\mathrm{n}}$, where $\mathrm{n}$ is slightly superlinear, $1<\mathrm{n}<1.3$. The behaviour at low temperatures is believed to be due to thermally activated local motions, presumably of ions, and can be explained in terms of motion within asymmetric double-well potentials [7]. We will not consider this behaviour further here, since our main interest is in the effect of long-range diffusive motion of ions on the SLR rate.

Such diffusive ionic motion gives rise to fluctuating magnetic fields which can cause NSR via a variety of nuclear-spin interactions, e.g. chemical shift, dipolar or quadrupolar. A proper understanding of the SLR behaviour can thus only be achieved via a knowledge of the underlying microscopic transport mechanism for the dynamic ionic behaviour. It is also essential to understand the apparently universal aspects of the SLR behaviour; for example why always does $\alpha \approx 0.5$ (at non-negligible ion concentrations)?

\section{Models for NSR in ionic glasses}

The first model for ionic-motion-mediated NSR was proposed by Bloembergen, Purcell and Pound (BPP) [8]. By assuming that the time-dependent correlation function, $g(t)$, characterising the motion between interacting nuclei was of a simple exponential form, with a single correlation time $\tau$ at a given temperature, a Lorentzian expression for $\mathbf{T}_{1}$ was obtained [8]:

$$
1 / \mathrm{T}_{1}=\mathrm{k}\left[\tau /\left(1+\omega^{2} \tau^{2}\right)+4 \tau /\left(1+4 \omega^{2} \tau^{2}\right)\right]
$$

where $\mathrm{k}$ is a constant. If $\tau$ is assumed to be thermally activated, with activation energy $\mathrm{W}$, the BPP model predicts that a plot of SLR rate versus inverse temperature should be symmetrical with activation energies $+W$ and $-W$ on the high- and low-temperature sides of the peak, respectively. Further, the BPP model predicts that the SLR rate should be independent of the Larmor frequency on the high-T side of the peak, but $\propto \omega_{L}{ }^{-2}$ on the low-T side. Evidently, this simple model fails to account for the experimental behaviour exhibited by ionically conducting glasses.

A number of suggestions have been made in an attempt to explain the non-BPP-like NSR behaviour. For example, it has been proposed [9] that the discrepancy arises because, whereas the d.c. conductivity is determined by long-range ionic migration, NSR could be sensitive to more short-range motions, for which the energetics of ionic transport might be different. Various empirical modifications of the BPP expression (eqn. 2) have also been proposed [4,10], as has the use of the empirical stretchedexponential (Kohlrausch-Williams-Watts) function for the correlation function $g(t)[4$, 12], but these approaches all suffer from the disadvantage that they lack a microscopic physical basis. The "coupling model [11] can be said to have a microscopic physical basis, but it is not yet founded on rigorous theoretical grounds.

Recently, Funke and coworkers [13] have applied a "jump-diffusion" model [14] for ionic motion to the problem of NSR in glasses. Although this theory predicts the correct qualitative behaviour of the SLR rate, viz. an asymmetric peak of $\mathrm{T}_{1}^{-1} \mathrm{vs} . \mathrm{T}^{-1}$, it is not clear whether use of this model is valid in the case of (super)ionic glasses since, firstly, it is based on Debye-Hückel theory (strictly only valid for very low ion concentrations), and secondly it is not clear whether the discrete forward and reverse 
hopping events assumed in this approach are an appropriate description of the microscopic ionic transport mechanism.

\section{The diffusion-controlled relaxation model}

Since all approaches proposed to date suffer, in our view, from various drawbacks, we have therefore recently developed a theory for NSR in disordered systems which is as simple as possible and yet is believed to capture all the essential physics. This model has been described in detail elsewhere $[5,15]$ but, for convenience, we summarise here the salient features.

The theory is based on the diffusion-controlled relaxation (DCR) model, previously applied to the case of electrical relaxation in ionic glasses $[16,17]$, in which it is assumed that the ionic relaxation events, giving rise to the dispersive dynamic ion-transport behaviour, occur in a series fashion: an ion at a given site can only relax if it is "triggered" by the arrival of another diffusing ion at the site. Such a process is one of "target diffusion" [18], and the microscopic transport mechanism can be recognised as involving interstitialcies [16].

The correlation function, $g(t)$, describing this triggered relaxation, when all diffusing ions, assumed to be randomly spatially situated and diffusing in $3 \mathrm{D}$, can act as triggering entities, is $[17,19]$ :

$$
g(t)=c \exp \left(-t / \tau^{\prime}\right) \exp \left[-(t / \tau)^{\frac{1}{2}}\right]
$$

where $c$ is a constant $(0<c<1)$, and the two characteristic correlation times $\tau$ and $\tau^{\prime}$ are given by

$$
1 / \tau^{\prime}=4 \pi \mathrm{DRn}
$$

and

$$
1 / \tau=64 \pi \mathrm{DR}^{4} \mathrm{n}^{2}
$$

where $D$ is the diffusion coefficient of the mobile ions, $n$ is their concentration, $R$ is the radius of the (interstitialcy) site within which the relaxation event is supposed to occur (or equivalently the effective interaction radius between triggering and relaxing ions). It is assumed in eqn. (4) that ionic relaxation in the absence of triggering events, arising say from the local motion of ions by thermal excitation over an energy barrier $W$ from a stable to a metastable position in a double-well potential, does not occur. The constant $c$ in eqn. (3) is associated with the instantaneous relaxation events caused by triggering ions being inside the interaction radius $R$ at time $t=0$. This unphysical feature can be removed by assuming that a diffusing triggering ion induces a finite rate of relaxation of a target instead of instantaneous triggering, in which case $g(t)$ assumes a simple exponential form at very short times, changing smoothly to the form of eqn. (3) at longer times [20]. Note that eqn. (3) can be rewritten in the form of a stretched exponential, albeit with a time-dependent exponent $\beta$ [17]. 


\section{Application of the DCR model to NSR in ionic glasses}

If NSR of a particular nucleus of an ion at a given site in a glassy matrix is caused by the fluctuating fields due to the diffusive motion of other ions, it is evident that NSR can only occur when a diffusing ion approaches another ion sufficiently closely for the fluctuating fields to be large enough to induce relaxation. Thus, the process is inherently one of target diffusion, and it is a natural extension of the DCR model to apply it to the case of NSR [5, 15].

The expression for the SLR rate can be calculated from the spectral density function, $J(\omega)$, obtained by a Laplace transform of the correlation function $g(t)$ (eqn. 3), where

$$
1 / \mathrm{T}_{1}=\mathrm{C}[\mathrm{J}(\omega)+4 \mathrm{~J}(2 \omega)]
$$

and where $C$ is a constant. The expression for $J(\omega)$ obtained in this way [5] is a complicated, complex function for which the real part (and hence $T_{1}$ ) can only be obtained by numerical means [17].

However, the expression for $J(\omega)$, and hence also that for the SLR rate, is the sum of two terms, the first of which is simply the BPP expression (eqn. 2), and the non-BPP-like behaviour therefore arises from the second term. This correction term tends to zero, i.e. BPP-like behaviour is recovered, for two conditions:

i) for $\omega \tau^{\prime}<<1$, so that the high-temperature, Larmor-frequency-independent region of the peak of $T_{1}^{-1}$ versus $1 / T$ (where $\omega \tau^{\prime}<<1$ ) is unaffected by non-exponential behaviour;

(ii) $\quad$ for $\left(\tau^{\prime} / \tau\right)^{\frac{1}{2}}=4\left(R^{3} n\right)^{\frac{1}{2}} \rightarrow 0$.

Thus, from (ii), the non-BPP-like correction term to the SLR rate is predicted to become negligible as the ion concentration tends to zero (see sect. 7).

\section{Temperature dependence of the SLR rate}

The expression for the SLR rate, obtained from the DCR model [5], is temperature dependent because both $\tau$ and $\tau^{\prime}$ are temperature dependent and, in an Arrhenius plot of $T_{1}-1$, a peak is predicted when the condition $\omega \tau^{\prime}=\gamma$, where $\gamma$ is a constant of order unity [21]. From eqn. (4) $\tau^{\prime} \propto 1 / \mathrm{Dn}$, and hence $\tau^{\prime} \propto 1 / \sigma$. Thus, for a fixed Larmor frequency, the peak in the SLR rate appears at a lower temperature the higher is the d.c. conductivity. The peak occurs at $1 / T=\left(k / E_{\sigma}\right) \ln \left(\gamma / \omega \tau_{0}^{\prime}\right)$ and hence is determined mainly by the d.c. conductivity activation energy. The peak temperature can be used to obtain an estimate for R. For $0.56 \mathrm{Li}_{2} \mathrm{~S}$. $0.44 \mathrm{SiS}_{2}$ the SLR data [22] give $\tau_{0}^{\prime}=1.5 \times 10^{-13_{\mathrm{S}}}$; note, however, that this is not to be interpreted as an inverse phonon frequency, but from eqn. (4) the DCR model predicts that $\tau_{0}^{\prime}=\mathrm{e}^{2} / 4 \pi \mathrm{k} \mathrm{T}_{\max } \sigma_{0} R$. Thus, from experimentally determined quantities $\left(T_{\max }, E_{\sigma}, \sigma_{0}\right)$, the interaction radius is estimated to be $R=0.7 \AA$.

On the high-T side of the peak of $T_{1}^{-1}$ vs. $1 / T$ (where $\omega \tau^{\prime}<<1$ ), BPP-like behaviour dominates and $T_{1}^{-1} \propto \tau^{\prime}$ in this region. Thus, the temperature dependence of $T_{1}$ is controlled by that of the ionic d.c. conductivity and hence the activation energy $E_{1}=E_{\sigma}$, as observed experimentally $[22,23]$. 
On the low-temperature side of the peak of SLR rate (where $\left.\omega \tau^{\prime}>>1\right)$, depending on the magnitude of the ratio $\left(\tau^{\prime} / \tau\right)$, the non-BPP-like correction term to $J(\omega)$ and hence $T_{1}$ may become significant, in which case the low-T activation energy $E_{2}$ is appreciably smaller than $\mathrm{E}_{1}$ [5]. In the DCR theory of NSR, the limiting value of the ratio of low-T and high-T activation energies is $\alpha=E_{2} / E_{1}=0.5$ [5]. This is a general feature of targetdiffusion theories [24] and arises because of the $t^{\frac{1}{2}}$ time dependence characteristic of diffusive processes; this therefore explains the widespread experimental observation that $\alpha \approx 0.5[5]$.

\section{Ionic concentration dependence of the SLR rate}

As mentioned in sect. 5, the deviation from BPP-like behaviour of the SLR rate in the DCR model is predicted to be a function of (mobile) ion concentration, $n$; the behaviour reverts to BPP-like as $n^{\frac{1}{2}}$ tends to zero. $[5,15]$. The physical reason for this compositional behaviour is that a simple exponential form for the correlation function, $\mathrm{g}(\mathrm{t})$, (i.e. BPP-like behaviour) is recovered when there is no influence on the probability of a site being relaxed due to the occurrence of previous diffusion-triggered relaxation events, i.e. when there is no correlation between relaxation events [19], viz. in the limit of very small ion concentrations.

Experimental data for the compositional dependence of the (temperature-dependent) behaviour of the SLR rate have been obtained for alkali germanate glasses ( $\mathrm{Li}:[6,7] ; \mathrm{Na}$ : [25]); BPP-like behaviour $\left(E_{2} \rightarrow E_{1}\right)$ is observed in the limit of very low ion concentrations. The behaviour predicted by the DCR model for NSR $[5,15]$ is in accord with that observed experimentally, although there is some uncertainty associated with the fact that the experimental data are a function of total ion concentration, $N$, whereas the predictions of the DCR model are in terms of the mobile ion concentration, $n$; it is presently not clear what is the relationship between $\mathrm{n}$ and $\mathrm{N}$. It should be noted, however, that the sub-linear power-law dependence of the SLR time on Larmor frequency, $\mathrm{T}_{1} \propto \omega^{\mathrm{m}}(\mathrm{m} \leq 1)$, observed experimentally in such (poorly-conducting) oxide glasses [25] is not consistent with the predictions of the DCR model in its simplest form $(m \geq 1.5)$. However, on the basis of the very limited amount of experimental data available for superionic glasses (see e.g. ref. [22]), it appears that the frequency dependence of $T_{1}$ in such cases $\underline{\text { is }}$ consistent with the DCR model.

\section{NSR and the mixed-alkali effect}

The mixed-alkali effect (MAE) refers to the markedly non-linear changes in ionic transport behaviour when one type of conducting ion in a glass is partially substituted by another type (see ref. [3] for a review of the MAE). For example, the ionic d.c. conductivity typically decreases by 5-6 orders of magnitude upon ion substitution and passes through a minimum at approximately the equimolar ion concentration; this behaviour results primarily from a corresponding maximum in the d.c. conductivity activation energy, $\mathrm{E}_{\sigma}$. However, the cause of the MAE is presently unclear, principally because a detailed understanding of the mechanism of ionic transport in glasses is lacking. Thus, it is not clear whether the MAE is due primarily to changes in ionic mobility $\mu$ or mobile ion concentration $n$, or a combination of the two. In those systems where it is feasible, radioactive tracer diffusion measurements of the individual diffusion coefficients, D, of conducting ions in mixed-alkali glasses have shown that $\mathrm{D}$ for the majority ionic species always decreases with increasing substitution of the minority species (see, e.g. ref [26]). Thus, according to the Nernst- 
Einstein relation, where $\mathrm{D} \propto \mu$, the ionic mobility of the majority species also decreases monotonically with increasing substitution of the minority ion, leading to a cross-over in diffusion coefficients at some intermediate mixed-ion concentration. However, such experiments do not allow information to be obtained on the concomitant behaviour of the mobile-ion concentration, $\mathrm{n}$.

In principle, measurements of the temperature-dependent SLR behaviour of mixedalkali glasses should shed light on this problem since, according to the DCR model, the asymmetry of the peak of SLR rate versus inverse temperature (for example as measured by the ratio of low- and high-temperature activation energies, $E_{2} / E_{1}$ ) is determined by the ratio of correlation times, $\left(\tau^{\prime} / \tau\right) \propto n$ (c.f. eqn. 4,5$)$. Previous NSR studies on mixed-alkali glasses [10] were confined to a very limited number of compositions. Therefore, we have recently undertaken an extensive study of the SLR behaviour of the MAE in a number of compositions of mixed $\mathrm{Na}-\mathrm{Li}$ disilicate glasses $[27]$.

The variation of the activation energy, $E_{2}$, on the low-temperature side of the peak of the ${ }^{7 \mathrm{Li}}$ SLR rate as a function of inverse temperature as a function of $\mathrm{Na}$ substitution is shown in fig. 1, together with the corresponding variation of the d.c. conductivity activation energy, $E_{\sigma}$. The resultant variation in the ratio of activation energies, $\alpha=$ $\mathrm{E}_{2} / \mathrm{E}_{\sigma}$ is shown in fig. 2, together with the two data points due to Göbel et al [10]. It can be seen that, within experimental error, this ratio is constant with alkali substitution, and $\alpha \approx 0.4$.

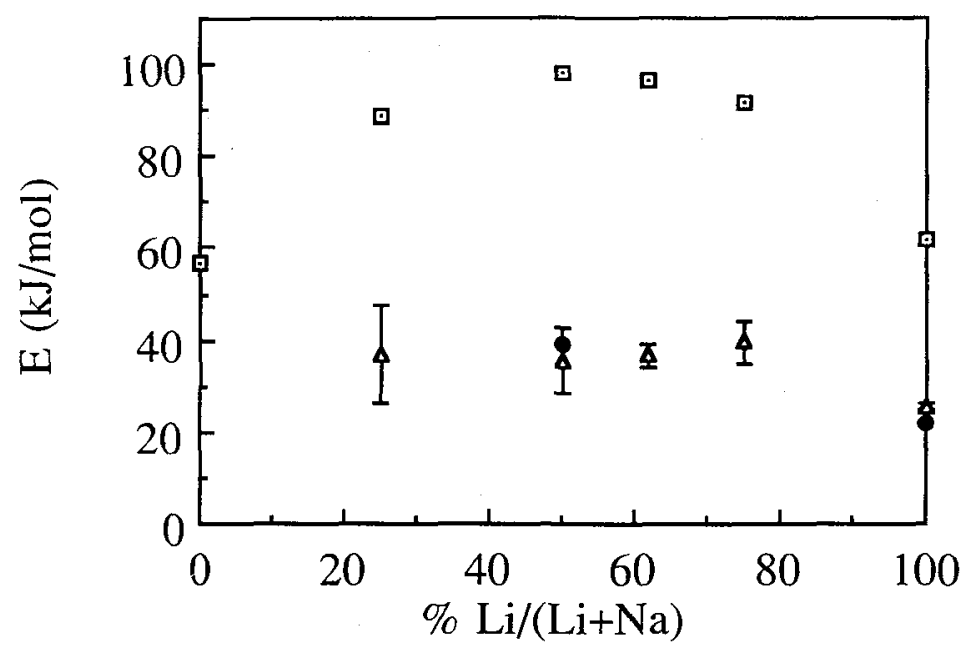

Fig. 1 Variations in the activation energies for ${ }^{7} \mathrm{Li}$ spin-lattice relaxation in the lowtemperature regime, $E_{2}$, measured in this work $(\Delta ;[27])$ and by Göbel et al [10] ( $\left.\bullet\right)$, and of the d.c. conductivity activation energy, $\mathrm{E}_{\sigma}$, measured by Tomazawa and Yoshiyagawa [31] ( $\square$ ), as a function of $\mathrm{Na}$ substitution in mixed (Na-Li) disilicate glasses. 
The result shown in fig. 2 is clear evidence that the MAE (at least in the Na-Li silicate glasses studied here) is not due to a marked decrease in the concentration of mobile ions when two alkali species are mixed, as predicted for defect-based mechanisms involving pairing of unlike ions [28] or by the weak-electrolyte theory [29]. Instead, it appears that the MAE arises predominantly from changes in ionic mobility, presumably due to changed inter-ionic interactions, although the recent diffusion-limited percolation mechanism of Bunde et al [30] may also possibly play a role.

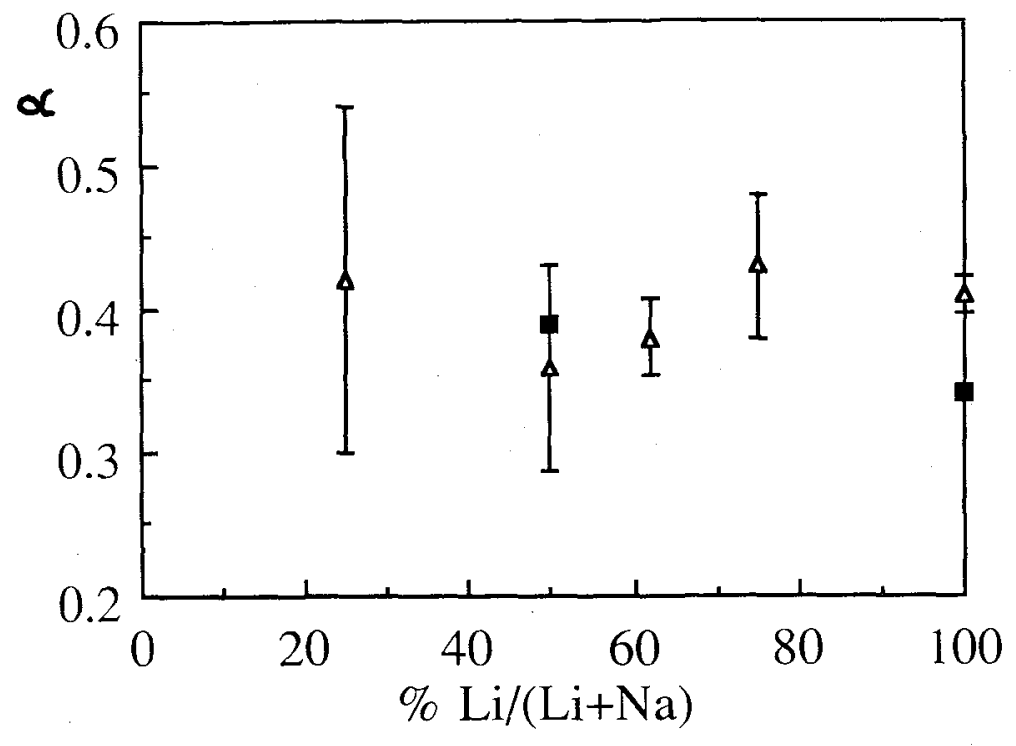

Fig. 2 Variation of the ratio of activation energies of the low-temperature ${ }^{7} \mathrm{Li}$ spinlattice relaxation rate [27] and of the d.c. conductivity [31], viz $\alpha=E_{2} / E_{\sigma}$, as a function of Na substitution in mixed (Na-Li) disilicate glasses $(\Delta)$. The two data points from Göbel et al [10] are also shown (where the $E_{\sigma}$ values used were their own, not those from ref. [31]).

However, some caution has to be exercised in this interpretation concerning the likely magnitude of changes in the mobile-ion concentration that could be inferred by monitoring changes in $\alpha=E_{2} / E_{\sigma}$. A study $[6,7]$ of the ion concentration dependence of the single alkali glass $\left(\mathrm{Li}_{2} \mathrm{O}\right)_{x}\left(\mathrm{GeO}_{2}\right)_{1-\mathrm{x}}$ has shown that $\alpha$ reaches a limiting value $(\approx 0.4)$ for $x \geq 0.1$; below this composition, $\alpha$ increases rapidly towards the value of unity (see sect. 7). From an examination of the ${ }^{7} \mathrm{Li} \mathrm{T}_{1}$ data for the mixed $\mathrm{Na}-\mathrm{Li}$ disilicate glass shown in fig. 2, it can be seen that there is no apparent increase in $\alpha$ at the $50: 50$ mixedalkali composition, and certainly there is no evidence that $\alpha$ tends to approach unity at this composition. Thus, from the predictions of the DCR model, and from the experimental composition-dependent data for $\alpha$ due to Kanert et al $[6,7]$, it is reasonable to state that the change in mobile-ion concentration with increasing substitution of $\mathrm{Li}$ by $\mathrm{Na}$ in ( $\mathrm{Li}-\mathrm{Na}$ ) disilicate is probably not more than a factor of $3-5$; certainly, the mobile-ion concentration does not appear to decrease significantly below $\approx 10 \%$ of the 
value characteristic of the single-alkali compositions, otherwise a marked increase in $\alpha$ should occur, as observed in the results for the single glasses $[6,7]$.

\section{Acknowledgements}

I am grateful to the Nuffield Foundation for the award of a Science Research Fellowship and to the CNRS for financial support.

\section{References}

[1] Ravaine D. and Souquet J.L., Phys. Chem. Glasses 18 (1977) 27.

[2] Anderson O.L. and Stuart D.C., J. Am. Ceram. Soc. 37 (1954) 573

[3] Ingram M.D., Phys. Chem. Glasses 28 (1987) 215.

[4] Martin S.W., Mat. Chem. Phys. 23 (1989) 225

[5] Elliott S.R. and Owens A.P., Phys. Rev. B44 (1991) 47.

[6] Estalji S., Kanert O., Steinert J., Jain H. and Ngai K.L., Phys. Rev. B43 (1991) 7481.

[7] Kanert O., Kloke M., Küchler, Rückstein S. and Jain H., Ber. Bunsenges. Phys. Chem. 95 (1991) 1061.

[8] Bloembergen N., Purcell E.M. and Pound R.V., Phys. Rev. 73 (1948) 679.

[9] Martin S.W., Bischof H.J., Mali M., Roos J. and Brinkmann D., Solid State Ionics, 18-19 (1986) 421.

[10] Göbel E., Muller-Warmuth W., Olyschlager H. and Dutz H., J. Mag. Res. 36 (1979) 371.

[11] Balzer-Jöllenbeck G., Kanert O., Jain H. and Ngai K.L., Phys. Rev. B39 (1989) 6071.

[12] Chung S.H., Jeffrey K.R., Stevens J.R. and Börgesson L., Phys. Rev. B41 (1990) 6154.

[13] Funke K. and Wilmer D., Europhys. Lett. 12 (1990) 363.

[14] Funke K., Solid State Ionics 28-30 (1988) 100.

[15] Elliott S.R. and Owens A.P., Ber. Bunsenges. Phys. Chem. 95 (1991) 987.

[16] Elliott S.R. Solid State Ionics, 27 (1988) 131.

[17] Elliott S.R. and Owens A.P., Phil. Mag. B60 (1989) 777.

[18] Glarum S.H., J. Chem. Phys. 33 (1960) 639.

[19] Bordewijk P., Chem. Phys. Lett. 32 (1975) 592.

[20] Condat C.A., Z. Phys. B77 (1989) 313.

[21] Lindsey C.P. and Patterson G.D., J. Chem. Phys. 73 (1980) 3348.

[22] Martin S.W., Patel H.K., Borsa F. and Torgeson D., J. Non-Cryst. Sol. 131-133 (1991) 1041.

[23] Pradel A. and Ribes M., J. Non-Cryst. Sol. 131-133 (1991) 1063.

[24] Kimmich R. and Voigt G., Z. Naturforsch. 33a (1978) 1294.

[25] Ngai K.L., Mundy J.N., Jain H., Kanert O. and Balzer-Jöllenbeck G., Phys. Rev. B39 (1989) 6169.

[26] Jain H., Peterson N.L. and Downing H.L., J. Non-Cryst. Sol. 55 (1983) 283.

[27] Williams R.D. and Elliott S.R., Submitted to J. Non-Cryst. Sol.

[28] Ingram M.D., J. Am. Ceram. Soc. 63 (1980) 248.

[29] Kone A., Reggiani J.C. and Souquet J.L., Solid State Ionics, 9-10 (1983) 709.

[30] Bunde A., Maas P., Ingram M.D. and Ngai K.L., J. Phys. A24 (1991) L881; Bunde A., Maas P. and Ingram M.D., Ber. Bunsenges. Phys. Chem. 95 (1991) 977.

[31] Tomazawa M. and Yoshiyagawa M., Glastech. Ber. 56K (1983) 939. 